\title{
Stability of the (two-loop) Renormalization Group Flow for Nonlinear Sigma Models
}

Christine Guenther (guenther@pacificu.edu)

Department of Mathematics and Computer Science, Pacific University, Forest Grove, Oregon, 97116, USA

Todd A. Oliynyk (todd.oliynyk@sci.monash.edu.au)

School of Mathematical Sciences, Monash University, VIC 3800, Australia

\begin{abstract}
We prove the stability of the torus, and with suitable rescaling, hyperbolic space under the (two-loop) renormalization group flow for the nonlinear sigma model. To prove stability we use similar techniques to [10, where the stability of the torus under Ricci flow was first established. The main technical tool is maximal regularity theory.
\end{abstract}

Keywords: Renormalization Group flow, Ricci flow, nonlinear stability

Mathematics Subject Classifications (2000): 35K55, 53C80, 58Z05, 81T17

\section{Introduction}

The renormalization group flow equations for the world-sheet nonlinear sigma models arise from quantizing the classical action

$$
S(x)=\frac{1}{4 \pi \alpha^{\prime}} \int_{\Sigma} \gamma^{\alpha \beta} g_{i j}(x) \partial_{\alpha} x^{i} \partial_{\beta} x^{j} d V(\gamma)
$$

where $\alpha^{\prime}>0$ is the string coupling constant, $(\Sigma, \gamma)$ is a 2-dimensional Riemannian manifold (i.e. world sheet), $(M, g)$ is a $n$-dimensional Riemannian manifold (i.e. target space), and $x: \Sigma \rightarrow M ;\left(\theta^{1}, \theta^{2}\right) \mapsto\left(x^{1}(\theta)\right.$ $\left., \ldots, x^{n}(\theta)\right)$ is a map. To (perturbatively) quantize the classical theory, a momentum cutoff $\Lambda>0$ must be introduced. This gives rise to a one parameter family of quantum field theories indexed by the cutoff $\Lambda$. The target space metric becomes $\Lambda$ dependent and plays the role of the "coupling constants". The requirement that the one parameter family of field theories be equivalent on length scales $L \gg 1 / \Lambda$ leads to the Renormalization Group (RG) flow equations

$$
\frac{\partial}{\partial \Lambda} g_{i j}=-\beta_{i j}^{g} \text {. }
$$

In the perturbative regime $\left(\alpha^{\prime} \ll 1\right)$, the $\beta$-functions $\beta_{i j}^{g}$ can be expanded in powers of $\alpha^{\prime}[\underline{8}, 13$ :

$$
\beta_{i j}^{g}=\alpha^{\prime} R_{i j}+\frac{\alpha^{\prime 2}}{2} R_{i k l m} R_{j}^{k l m}+\mathrm{O}\left(\alpha^{\prime 3}\right) .
$$

(c) 2018 Springer Science+Business Media, Inc. Manufactured in The Netherlands. 
Here we are using $R_{i j k \ell}=g_{\ell m} R_{i j k}^{m}$ where $R_{i j k}^{\ell}=\partial_{i} \Gamma_{j k}^{\ell}-\partial_{j} \Gamma_{i k}^{\ell}+$ $\Gamma_{j k}^{m} \Gamma_{i m}^{\ell}-\Gamma_{i k}^{m} \Gamma_{j m}^{\ell}$. Introducing "time" by $t=-\ln (\Lambda)$, the RG flow equations become

$$
\frac{\partial}{\partial t} g_{i j}=-\alpha^{\prime} R_{i j}-\frac{\alpha^{\prime 2}}{2} R_{i k l m} R_{j}^{k l m}+\mathrm{O}\left(\alpha^{\prime 3}\right) .
$$

Truncating at the first order in $\alpha^{\prime}$ gives

$$
\frac{\partial}{\partial t} g_{i j}=-\alpha^{\prime} R_{i j}
$$

In the perturbative regime, the first order truncation should provide an acceptable approximation to the full RG flow. However, this is difficult to quantify as a rigorous definition of the $\beta$-functions requires a nonperturbative quantization of the nonlinear sigma model, and this has not yet been shown to exist. As has been noted previously [16], the first order truncation (3) is Ricci flow. It is easy to see (e.g. by considering the sphere) that there exist many solutions to Ricci flow which become singular in finite time. In [11] Hamilton showed that at a finite singular time $T<\infty$, the Riemannian curvature blows up, i.e. $\lim _{t \nearrow T}|R m|_{g}=$ $\infty$. Therefore for times near a singular time for Ricci flow, from the perturbative expansion for the beta-functions (2), it appears that the second order correction term would dominate even for $\alpha^{\prime} \ll 1$. This would signal a regime change where the first order truncation (i.e. Ricci flow) is no longer a valid approximation for the full RG flow. This suggests the strategy of using the second order (two-loop) truncation

$$
\frac{\partial}{\partial t} g_{i j}=-\alpha^{\prime} R_{i j}-\frac{\alpha^{\prime 2}}{2} R_{i k l m} R_{j}{ }^{k l m}
$$

as a measure of the error in approximating the full RG flow (3) by Ricci flow (44). A related motivation for studying (5), as discussed in [16], is that in certain situations, it is enough to consider the second order RG equations (5) to establish the existence of a continuum limit 9]. Finally, we note that it is tempting to view the higher order truncations of the $\mathrm{RG}$ flow equations (3) as natural modifications of Ricci flow. In this light, the $\beta$-function expansion (2) in powers of $\alpha^{\prime}$ generates specific modifications to Ricci flow. As with other equations that arise from physical considerations, these equations may have "nice" properties, which we think, at the very least, is worth investigating. However, we note that whereas (5) can be studied using parabolic techniques (at least for $\alpha^{\prime}$ small enough), the third and higher order correction would involve polynomials of the curvature and its derivatives 13. This would mean that in trying to study the third and higher order equations as evolution equations, some form of the Nash-Moser implicit 
function theorem would be necessary to overcome the resulting loss of derivatives.

As a first step in the analysis of the second order RG flow (5), we prove the stability of the flat torus, and with suitable rescaling hyperbolic space, under the flow. See sections 2 and 3 for the theorems and proofs. The main theorems are Theorem 1 and Theorem 2 .

\section{Stability of the second order RG flow at $M=\mathbb{T}^{n}$.}

Let $\bar{g}$ be a flat Riemannian metric on a closed $n$-dimensional manifold $M$. In this section we show that there exists a neighborhood of $\bar{g}$ in an appropriate function space such that if $g_{0}$ is in this neighborhood, then the solution $g(t)$ of the renormalization group flow with $g(0)=g_{0}$ converges exponentially quickly to a centermanifold at $\bar{g}$ consisting entirely of flat metrics. To do so we will apply maximal regularity theory, as introduced by [4], and applied to fully nonlinear equations in [5]. See [21] for an introduction to these methods in a geometric setting. The argument is similar to that used to show the stability of the Ricci flow at flat metrics [10, and at hyperbolic metrics [14]1; however, the second order renormalization group flow is no longer quasilinear, and so results for fully nonlinear equations must be used (see the crosscurvature flow result in [14 for another fully nonlinear example). We will work with the nested little Hölder spaces of 2-tensors, denoted $h^{i+\alpha}$, which are the completion of the $C^{\infty} 2$-tensors in the Hölder norm $\|\cdot\|_{i+\alpha}$. Letting $E_{0}=h^{0+\sigma}, E_{1}=h^{2+\sigma}, X_{0}=h^{0+\rho}$, and $X_{1}=h^{2+\rho}$, where $0<\sigma<\rho<1$, one has

$$
X_{1} \subset E_{1} \subset X_{0} \subset E_{0},
$$

with $X_{0}$ and $X_{1}$ the continuous interpolation spaces required to apply maximal regularity theory (for an introduction to these spaces, their connection with the more familiar real interpolation spaces, and a justification of the above inclusion, see the beginning of Section 3.3 [10]). We prove the following theorem:

Theorem 1 (Stability of n-dimensional Torus). Let $\bar{g}$ be a flat Riemannian metric on a closed $n$-dimensional manifold $M, n \geqslant 3$, and let $g(t)$ satisfy the second order $R G$ flow

$$
\begin{aligned}
\frac{\partial}{\partial t} g & =-2 R c(g)-\frac{\alpha^{\prime}}{2} R m^{2} \\
g(0) & =g_{0} .
\end{aligned}
$$

\footnotetext{
${ }^{1}$ See 24] for results on the stability of constant nonzero curvature spaces under Ricci flow, using other means.
} 
There exists a ball $B_{r}(\bar{g}) \subset h^{2+\rho}, r>0$, such that if $g_{0} \in B_{r}(\bar{g})$, then the solution $g(t)$ of (6) converges exponentially quickly to a flat metric.

Proof. Following [6], in order to obtain a strictly parabolic equation, for any positive definite 2-tensors $g, u$ we define a vector field

$$
W_{u, g}^{i}=-g^{i j} u_{j k}^{-1} g^{k l} g^{p q}\left(\nabla_{p} u_{q l}-\frac{1}{2} \nabla_{l} u_{p q}\right),
$$

and a modified flow

$$
\frac{\partial}{\partial t} g=-2 R c(g)+L_{W_{u, g}} g-\frac{\alpha^{\prime}}{2} R m^{2}(g),
$$

where $L_{W_{u, g}} g$ is the Lie derivative of $g$ in the direction $W_{u, g}$. We shall call this the second order DeTurck-RG flow. If $g$ is a solution of (8), then $\phi_{t}^{*} g$ is a solution of ([6), where $\phi_{t}$ is the family of diffeomorphisms generated by integrating the vector field $-W_{u, g}$. When $u=\bar{g}$ we see that $\bar{g}$ is also a fixed point of (흐). By the standard first variation formula $\left.\frac{d}{d \varepsilon} R c(g+\varepsilon h)\right|_{\varepsilon=0}$ (see Theorem 1.174 [1]), the linearization of the first two terms of the right hand side at $\bar{g}$ is given by

$$
\left.D\left(-2 R c(g)+L_{W_{\bar{g}, g}} g\right)\right|_{g=\bar{g}} h=\bar{\Delta}_{L} h,
$$

where

$$
\bar{\Delta}_{L} h_{i j}=: \bar{\Delta} h_{i j}+2 \bar{R}_{k i j l} h^{k l}-g^{k l} \bar{R}_{i l} h_{k j}-g^{k l} \bar{R}_{j l} h_{i k}
$$

is the Lichnerowicz Laplacian with respect to the metric $\bar{g}$, and $\bar{\Delta}$ denotes the usual Laplacian with respect to $\bar{g}$ (see Chapter 3.3 in [2] for complete details of the calculation). We denote $\bar{R}_{i j k l}=R m(\bar{g})_{i j k l}$ and $\bar{R}_{i j}=R c(\bar{g})_{i j}$. So letting $u=\bar{g}$, the linearization of (8) at a flat metric $\bar{g}$ is

$$
\frac{\partial}{\partial t} h=\bar{\Delta} h=: A_{\bar{g}} h
$$

The existence of an exponentially attractive centermanifold is a consequence of Theorem 3.3 in [5], and so we verify its hypotheses. It is convenient to rewrite equation (8) as

$$
\frac{\partial}{\partial t} g=A_{\bar{g}} g(t)+G(g(t))
$$

where $A_{\bar{g}}$ is the linearization of the right hand side of (8). The hypotheses of the theorem are:

$A_{\bar{g}}: X_{1} \rightarrow X_{0}$ is sectorial (and extends to sectorial $A_{\bar{g}}: E_{1} \rightarrow E_{0}$ ), $G \in C^{1}\left(O, X_{0}\right)$, where $O \subset X_{1}$ is a neighborhood of $\bar{g}$,

$G(\bar{g})=0, \quad G^{\prime}(\bar{g})=0$. 
We recall that an operator being sectorial roughly means that the spectrum is bounded in a wedge in a left half plane, and its resolvent is uniformly bounded on the comlementary right half plane. By definition, the space $h^{2+\alpha}$ consists of 2-tensors whose second derivatives are in $h^{0+\alpha}$; as was shown in Lemma 3.4 [10], by standard Schauder estimates the operator $A_{\bar{g}}: X_{1} \rightarrow X_{0}$ is sectorial; continuous Frechet differentiability of $G$ and the existence of $O$ can be checked as in Section 4 of [14. Since

$$
\left\langle A_{\bar{g}} h, h\right\rangle=\int\langle\bar{\Delta} h, h\rangle=-\|\bar{\nabla} h\|^{2}
$$

the kernel of $A_{\bar{g}}$ consists of all parallel 2-tensors, and is an $\frac{n(n+1)}{2}$ dimensional subspace of the tangent space of symmetric 2-tensors.Therefore the hypotheses of the theorem are satisfied, and there is an $r>0$ such that if $g_{0} \in B_{r}(\bar{g}) \subset h^{2+\rho}$, there exists a centermanifold at $\bar{g}$ of dimension at most $\frac{n(n+1)}{2}$ that is exponentially attractive for times $t \leqslant \tau(r)$. Flat metrics are fixed points of the flow, and so since they are exponentially attracted to the centermanifold, they must lie on the centermanifold. Since the space of flat metrics on the torus is $\frac{n(n+1)}{2}$ dimensional, the centermanifold must consist precisely of flat metrics.

We can in fact obtain a global result, using Corollary 9.1.6 in [17] to obtain long term existence. Consider a solution $g$ of (8) . By definition (7), if $g$ converges exponentially to a flat metric, then $W_{\bar{g}, g}$ converges exponentially to zero. The curvature terms also converge exponentially to zero, and so the right hand side of (8) converges to zero exponentially and $\left|\frac{\partial}{\partial t} g\right| \leqslant C e^{-\omega t}$, where $\omega>0$. (See also the proof of Theorem 3.7 in [10].) We therefore have global existence and convergence to a flat metric.

We have shown exponential convergence of solutions of DeTurckRG flow, but we have exponential convergence of the second order RG flow as well. The idea is that if the norm of a vector field $W$ decays exponentially, then the diffeomorphisms $\phi_{t}$ generated by the vector field converge exponentially to a fixed diffeomorphism. (To see this just calculate the length of the integral curves. See also Lemma 3.5 and Proposition 3.6 in [10].) Since the solutions of (86) and (6) are related by the pullback $\phi_{t}^{*}$, the theorem is proved. 


\section{Stability of the modified second order RG flow at $M=\mathbb{H}^{n}$}

We next consider the stability of a closed hyperbolic manifold. Let $\bar{g}$ be a Riemannian metric of constant negative curvature $K$ on a closed $n$-dimensional manifold $M$ with $n \geqslant 3$. In this case $\bar{g}$ is not a fixed point of the second order RG flow (6), and so we will instead consider an equation that is related to the renormalization group flow only by diffeomorphism and rescaling. To proceed, let $g$ satisfy the original RG flow

$$
\frac{\partial}{\partial t} g=-2 R c(g)-\frac{\alpha^{\prime}}{2} R m^{2}(g)
$$

for $0<t<T$. For a constant $c>0$, let $\sigma(t)=c(t+1)$ and $\tau(t)=$ $\frac{1}{c} \log (t+1)$, so that $\frac{\partial}{\partial t} \tau=\frac{1}{c(t+1)}$. Define $\hat{g}(\tau)$ by

$$
g(t)=\sigma(t) \phi_{t}^{*} \hat{g}(\tau(t))
$$

where $W$ is the vector field (7), and $\frac{\partial}{\partial t} \phi_{t}=-W_{u, \hat{g}}$. Then

$$
\begin{aligned}
\frac{\partial}{\partial t} g & =\frac{\partial}{\partial t}\left(\sigma(t) \phi_{t}^{*} \hat{g}(\tau(t))\right. \\
& =c \phi_{t}^{*}\left(\hat{g}(\tau(t))+c(t+1)\left[\phi_{t}^{*}\left(\frac{\partial \hat{g}}{\partial \tau} \frac{\partial \tau}{\partial t}\right)+\left.\frac{\partial}{\partial s}\right|_{s=0}\left(\phi_{t+s}^{*}(\hat{g}(\tau(t))]\right.\right.\right. \\
& =\phi_{t}^{*}\left(c \hat{g}(\tau(t))+\frac{\partial \hat{g}}{\partial \tau}-L_{W_{u, \hat{g}}} \hat{g}(\tau(t))\right) .
\end{aligned}
$$

Since

$$
\begin{aligned}
\frac{\partial}{\partial t} g & =-2 R c(g)-\frac{\alpha^{\prime}}{2} R m^{2}(g) \\
& =\phi_{t}^{*}\left(-2 R c(\hat{g})-\frac{\alpha^{\prime}}{2 c e^{c \tau}} R m^{2}(\hat{g})\right),
\end{aligned}
$$

$\hat{g}$ satisfies the evolution equation

$$
\frac{\partial}{\partial \tau} \hat{g}=-2 R c(\hat{g})+L_{W_{u, \hat{g}}} \hat{g}-\frac{\alpha^{\prime}}{2 c e^{c \tau}} R m^{2}(\hat{g})-c \hat{g},
$$

for $0 \leqslant \tau<\frac{1}{c} \log (T+1)$. This equation has a time-dependent coefficient $\frac{\alpha^{\prime}}{2 c e^{c \tau}}$, and so is no longer autonomous; however, since the coefficient decays exponentially we can handle this by considering instead the system

$$
\frac{\partial}{\partial \tau}\left(\begin{array}{c}
\hat{g}_{i j} \\
v
\end{array}\right)=\left(\begin{array}{c}
-2 \hat{R}_{i j}+L_{W_{u, \hat{g}}} \hat{g}_{i j}-v^{2} \hat{R}_{i k l m} \hat{R}_{j}^{k l m}-c \hat{g} \\
-\frac{c}{2} v
\end{array}\right) .
$$


Notice that we can explicity integrate the $v$ equation to get $v=v_{0} e^{-\frac{c}{2} \tau}$. This shows, in particular, that if $(\hat{g}(\tau), v(\tau))$ is a solution to (13), then $g(\tau)$ will solve (12) with $\alpha^{\prime}$ defined via the formula

$$
\alpha^{\prime}=2 c v_{0}^{2} .
$$

Since $\hat{g}=\bar{g}$ is a metric of constant curvature $K<0$, we observe that

$$
\begin{aligned}
\bar{R}_{i j k l} & =K\left(\bar{g}_{i l} \bar{g}_{j k}-\bar{g}_{i k} \bar{g}_{j l}\right), \\
\bar{R} m_{i j}^{2} & =2 K^{2}(n-1) \bar{g}_{i j}, \\
\bar{R}_{i j} & =K(n-1) \bar{g}_{i j} .
\end{aligned}
$$

The Lichnerowicz Laplacian is

$$
\bar{\Delta}_{L} h_{i j}=\bar{\Delta} h_{i j}+2 K H \bar{g}_{i j}-2 n K h_{i j},
$$

where $H=\bar{g}^{i j} h_{i j}$. Setting

$$
c=-2 K(n-1),
$$

we then get that $(\bar{g}, 0)$ is a fixed point for (13). By (9) and (14), it is clear that the linearization of (13) at $(\hat{g}, v)=(\bar{g}, 0)$ is given by

$$
\partial_{t}\left(\begin{array}{c}
h \\
w
\end{array}\right)=\left(\begin{array}{cc}
\bar{\Delta}_{L}-c & 0 \\
0 & -\frac{c}{2}
\end{array}\right)\left(\begin{array}{c}
h \\
w
\end{array}\right) .
$$

Letting $A_{(\bar{g}, 0)}(h, w)$ denote the righthand side of the linearization, we see that

$$
\begin{aligned}
\left\langle A_{(\bar{g}, 0)}(h, w),(h, w)\right\rangle & =\int(h, w)\left(\begin{array}{cc}
\bar{\Delta}_{L}-c & 0 \\
0 & -\frac{c}{2}
\end{array}\right)\left(\begin{array}{c}
h \\
w
\end{array}\right) \\
& =\int\left(\bar{\Delta}_{L}-c\right) h_{i j} h^{i j}-\frac{c}{2} w^{2} \\
& =\int\left(\bar{\Delta} h_{i j}+2 K H g_{i j}-2 n K h_{i j}-c h_{i j}\right) h^{i j}-\frac{c}{2} w^{2} \\
& =-\|\bar{\nabla} h\|^{2}+2 K\|H\|^{2}-(2 n K+c)\|h\|^{2}-\frac{c}{2}\|w\|^{2} .
\end{aligned}
$$

Using the Koiso Bochner formula [15]

$$
\|\bar{\nabla} h\|^{2}=\frac{1}{2}\|\bar{T}\|^{2}+\|\bar{\delta} h\|^{2}-n K\|h\|^{2}+K\|H\|^{2}
$$

where $\bar{T}_{i j k}=\bar{\nabla}_{k} h_{i j}-\bar{\nabla}_{i} h_{j k}$, yields

$$
\begin{aligned}
\left\langle A_{\bar{g}, 0}(h, w),(h, w)\right\rangle= & -\frac{1}{2}\|\bar{T}\|^{2}-\|\bar{\delta} h\|^{2}+K(n-2)\|h\|^{2}+K\|H\|^{2} \\
& +2 K(n-1)\|w\|^{2} .
\end{aligned}
$$


This shows that the spectrum of $A_{(\hat{g}, 0)}$ is strictly negative for $n \geqslant 3$. Since the Lichnerowicz Laplacian is self-adjoint and elliptic, the resolvent bound follows again from standard Schauder estimates (see e.g. Lemma 3.4 in [10]).

Thus, by Theorem 9.1.7 in [17], one has exponential convergence of (13) for initial data in an appropriate neighborhood of $(\bar{g}, 0)$. Since we have an explicit formula for $v$, letting $\alpha^{\prime}=2 c v_{0}^{2}$ we have proved the following theorem:

Theorem 2 (Stability of modified RG flow for n-dimensional hyperbolic space). Let $\bar{g}$ be a Riemannian metric of constant curvature $K<0$ on a closed $n$-dimensional manifold $M, n \geqslant 3$, and let $\hat{g}(\tau)$ satisfy the modified DeTurck second order $R G$ flow

$$
\begin{aligned}
& \frac{\partial}{\partial \tau} \hat{g}=-2 R c(\hat{g})+L_{W_{\bar{g}, \hat{g}}} \hat{g}(\tau)-\frac{\alpha^{\prime}}{2 c e^{c \tau}} R m^{2}(\hat{g})-c \hat{g}(\tau), \\
& \hat{g}(0)=\hat{g}_{0}
\end{aligned}
$$

where $c=-2 K(n-1)$. Then for sufficiently small $\alpha^{\prime}$, there exists a ball $B_{r}(\bar{g}) \subset h^{2+\rho}$ such that if $g_{0} \in B_{r}(\bar{g})$, then the solution $\hat{g}(\tau)$ of (16) exists for all time and converges exponentially quickly to a metric of constant negative curvature.

We have seen that $\hat{g}(\tau)$ converges to one of constant negative curvature, but what happens to the original solution of the second order RG flow? Recall that

$$
g(t)=\sigma(t) \phi_{t}^{*} \hat{g}(\tau(t))
$$

is a solution of the second order RG flow. The scaling factor $\sigma$ blows up, but the vector field $W_{\bar{g}, g}$ converges exponentially to zero. Therefore, one has the following corollary:

Corollary 1. Let $\bar{g}$ be a Riemannian metric of constant curvature $K<0$ on a closed $n$-dimensional manifold $M, n \geqslant 3$, and let $g(t)$ satisfy the second order $R G$ flow

$$
\begin{aligned}
\frac{\partial}{\partial t} g & =-2 R c(g)-\frac{\alpha^{\prime}}{2} R m^{2}(g) \\
g(0) & =g_{0},
\end{aligned}
$$

Then for sufficiently small $\alpha^{\prime}$, there exists a ball $B_{r}(\bar{g}) \subset h^{2+\rho}, r>0$, such that if $g_{0} \in B_{r}(\bar{g})$, then the solution $g(t)$ of (17) exists for all time and becomes more homogeneous in time. 


\section{Discussion}

We have established the stability of the torus, and with suitable rescaling hyperbolic space, under the second order (two-loop) RG flow (5). This shows that in a neighborhood of a flat, or with suitable rescaling a hyperbolic metric, the qualitative behavior of the first and second order RG flow equations are the same. In particular, this suggests that in these situations Ricci flow is a good approximation to the full RG flow. One immediate application of this result is to produce approximate solutions to the RG flow that involve order $\alpha^{\prime 2}$ corrections, and for which the Perelman type entropy proposed by Tseytlin [23] (see also [18, 19, 20]) is monotone, at least to order $\alpha^{\prime 2}$.

The results contained in this article are only a first step in the analysis of the second order RG flow (5). Clearly, it would be of interest to identify initial data which generate solutions for which the first and second order equations differ significantly. This could signal a breakdown of the perturbative regime and therefore would be of physical interest. It could also be of interest mathematically as the most likely mechanism for the breakdown would be the development of large curvature along the flow. Depending on the behavior of the solutions to the second order RG flow, this may (or may not) lead to new applications in differential geometry.

\section{Acknowledgements}

This work began while both authors were at the Albert-Einstein-Institute (AEI). We thank the AEI for its hospitality, and the director Gerhard Huisken of the Geometric Analysis and Gravitaton group for creating such a supportive and stimulating work environment.

\section{References}

1. Besse, A. Einstein manifolds, Springer Verlag, Heidelberg (1987)

2. Chow, Bennett; Knopf, Dan: The Ricci Flow: An Introcudtion, Mathematical Surveys and Monographs, Vol 110 (2004)

3. Cannarsa, Piermarco; Vespri, Vincenzo: Generation of analytic semigroups by elliptic operators with unbounded coefficients, SIAM J. Math. Analy. 18 (1987), no. $3,857-872$

4. Da Prato, Giuseppe; Grisvard, Pierre.: Equations d'évolution abstraites non linéaires de type parabolique, (French) Ann. Mat. Pura Appl. 120 (1979), 329396 
5. Da Prato, G.; Lunardi, A.: Stability, instability and center manifold theorem for fully nonlinear autonomous parabolic equations in Banach space, Arch. Rational Mech. Anal. 101 (1988), 115-141

6. DeTurck, Dennis M.: Deforming metrics in the direction of their Ricci tensors, improved version, Collected Papers on Ricci Flow, ed. H.-D. Cao, B. Chow, S.-C. Chu, and S.-T. Yau. Internat. Press, Somerville, MA, 2003

7. Ebin, David G.: The manifold of Riemannian metrics. 1970 Global Analysis (Proc. Sympos. Pure Math., Vol. XV, Berkeley, Calif., 1968) pp. 11-40 Amer. Math. Soc., Providence, R.I.

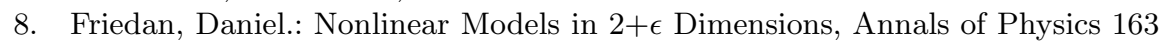
(1985), 318-419

9. Gawedzki, K.; Kupianinen, A.: Gross-Neveu model through convergent perturbation expansions, Commun. Math. Phys 102 (1985), 1-30

10. Guenther, Christine; Isenberg, James; Knopf, Dan.: Stability of the Ricci flow at Ricci-flat metrics, Comm. Anal. Geom. 10 (2002), 741-777

11. Hamilton, R.S.: Three-manifolds with positive Ricci curvature, J. Diff. Geom. 17 (1982), 255-306

12. Henry, Daniel: Geometric theory of semilinear parabolic equations. Lecture Notes in Mathematics, 840. Springer-Verlag, Berlin-New York, (1981).

13. Jack, I; Jones, D.R.T.; Mohammedi, N.: A four-loop caclulation of the metric $\beta$-function for the bosonic $\sigma$-model and the string effective action, Nuc. Phys. B322 (1989), 431-470

14. Knopf, Dan; Young, Andrea: Asymptotic stability of the cross curvature flow at a hyperbolic metric, arXiv: math.DG/0609767 v. 127 Sep 2006

15. Koiso, Norihito: On the second derivative of the total scalar curvature, Osaka J. Math. 16 (1979), 413-421

16. Lott, John: Renormalization Group Flow for General $\sigma$-Models, Commun. Math. Phys. 107 (1986), 165-176

17. Lunardi, Alessandra: Analytic semigroups and optimal regularity in parabolic problems. Progress in nonlinear differential equations and their applications 16, Birkhäuser Boston, Boston, MA, (1995)

18. Oliynyk, T.; Suneeta, V.; Woolgar, E.: Irreversibility of world-sheet renormaliation group flow, Phys. Lett. B 610 (2005), 115-121

19. Oliynyk, T.; Suneeta, V.; Woolgar, E.: A gradient flow for worldsheet nonlinear sigma models, Nucl. Phys. B 739 (2006), 441-458

20. Oliynyk, T.; Suneeta, V.; Woolgar, E.: A Metric for Gradient RG Flow of the Worldsheet Sigma Model Beyond First Order, Phys. Rev. D 76 (2007), 045001

21. Chow, Bennett et. al.: The Ricci Flow: Techniques and Applications Part III; preprint

22. Triebel, H.: Interpolation theory, function spaces, differential operators,(rev. ed.) Johann Ambrosius Barth Verlag, Heidelberg (1995)

23. Tseytlin, A.A.: On sigma model renormalization group flow, "central charge" action, and Perelman's entropy, Phys. Rev. D 75 (2007), 064024-6

24. Ye, R. Einstein metrics and Space Forms, Trans. Amer. Math. Soc. 338, no. 2 (1993), 871-896 